\title{
Aktuelle Infrastruktur im Labor für Gesteins- und Materialprüfung der Lehrstühle für Subsurface Engineering und für Bergbaukunde, Bergtechnik und Bergwirtschaft an der Montanuniversität Leoben
}

\author{
Christian Heiss ${ }^{1}$ und Jörg Krainz \\ 'Lehrstuhl für Bergbaukunde, Bergtechnik und Bergwirtschaft, Montanuniversität Leoben, Leoben, Österreich \\ ${ }^{2}$ Lehrstuhl für Subsurface Engineering, Montanuniversität Leoben, Leoben, Österreich
}

Eingegangen 28. Oktober 2017; angenommen 30. Oktober 2017; online publiziert 22. November 2017

\begin{abstract}
Zusammenfassung: Die aktuelle Infrastruktur, die dem Lehrstuhl für Subsurface Engineering und dem Lehrstuhl für Bergbaukunde, Bergtechnik und Bergwirtschaft im Impulszentrum für Rohstoffe (IZR) zur Verfügung steht, erlaubt qualitativ hochwertige Untersuchungen an mineralischen Rohstoffen, aber auch gesteinsähnlichen Werkstoffen. Eine Übersicht der wichtigsten Analysegeräte und Prüfanlagen soll das Leistungsvermögen des Labors widerspiegeln und potentiellen Kooperationspartnern aus Industrie und Wirtschaft als Anregungen für die Gestaltung zukünftiger Forschungsprojekte dienen.
\end{abstract}

Schlüsselwörter: Laborinfrastruktur, Probenvorbereitung, Materialprüfung, Festgestein, Lockergestein,

Versatzmaterial

Current State of Infrastructure in the Rock- and Material Testing Laboratory of the Chair of Subsurface Engineering and the Chair of Mining Engineering and Mineral Economics at the University of Leoben

Abstract: The current state of infrastructure in the laboratory of the Chair of Subsurface Engineering and the Chair of Mining Engineering and Mineral Economics at the Impulszentrum für Rohstoffe (IZR) provides the possibility of high quality testing of mineral raw materials, concrete, backfill material and soil. The overview of the most important testing equipment should show the lab's potential and give prospective project partners an idea of possible future research programmes.

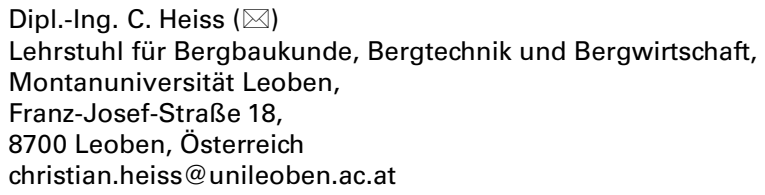

Keywords: Laboratory infrastructure, Sample preparation, Material testing, Hard rock, Loose rock, Backfill material

\section{Einleitung}

Am 21. Mai 2011 wurde im Beisein des damaligen Wirtschaftslandesrats Mag. Christian Buchmann und zahlreicher weiterer Gäste aus Politik, Forschung und Wirtschaft das Impulszentrum Rohstoffe (IZR) Leoben feierlich eröffnet. Das Gebäude wurde mitten im Campus der Montanuniversität Leoben in unmittelbarer Nähe zum "Impulszentrum für Werkstoffe" errichtet und bietet seitdem Wissenschaftlern und Forschern des Departments Mineral Resources Engineering auf einer Gesamtfläche von $3358 \mathrm{~m}^{2}$, wovon $1597 \mathrm{~m}^{2}$ als Laborfläche dienen, die Möglichkeit, mit modernsten Apparaturen und aktuellsten Methoden Untersuchungen an mineralischen Roh- und gesteinsähnlichen Werkstoffen durchzuführen [1].

Neben den Lehrstühlen für Gesteinshüttenkunde und Aufbereitung und Veredlung wird das Zentrum vorwiegend vom Lehrstuhl für Bergbaukunde, Bergtechnik und Bergwirtschaft und dem Lehrstuhl für Subsurface Engineering genutzt, wobei diesen zwei Lehrstühlen für wissenschaftliche Untersuchungen auf zwei unterirdischen Etagen gemeinsam Laborflächen von insgesamt etwa $370 \mathrm{~m}^{2}$ zur Verfügung stehen. Ergänzt werden die Laboreinrichtungen der beiden Lehrstühle durch eine Departmentswerkstätte - ausgerüstet unter anderem mit einer Drehbank, Standbohrmaschine, Bandsäge und einem Schweißplatz - und durch ein Probenlager, das auch vom Lehrstuhl für Aufbereitung und Veredlung genutzt wird.

Aufgrund der idealen räumlichen Voraussetzungen und der gemeinsamen Nutzung eines Großteils der Laborinfrastruktur konnte so im Impulszentrum Rohstoffe die Kooperation der Lehrstühle für Subsurface Engineering und Berg- 
baukunde, Bergtechnik und Bergwirtschaft intensiviert und der Wissenstransfer bezüglich Untersuchungs- und Prüfverfahren zwischen den verantwortlichen Mitarbeitern in den letzten Jahren weiter optimiert werden.

Die im Laborbereich neu eingerichteten Arbeitsplätze erforderten natürlich auch behördliche Genehmigungsverfahren. Diese verlangten nicht nur eine gründliche Auseinandersetzung mit den bis dato verwendeten Apparaturen was einige Neuanschaffungen bzw. Modernisierungen zur Folge hatte - sondern führten auch zur Ausarbeitung von Arbeits- und Prüfanweisungen, die in eine gemeinsame Laborordnung übernommen wurden. Die Laborordnung inklusive diverser Anweisungen und ein neuentwickeltes Probendokumentationssystem stellen derzeit einen wesentlichen Teil der Qualitätskontrolle einzelner Untersuchungsprozesse im Labor dar.

Obwohl derzeit eine Akkreditierung als Prüflabor weder von Seiten des Lehrstuhls für Bergbaukunde, Bergtechnik und Bergwirtschaft noch von Seiten des Lehrstuhls für Subsurface Engineering angedacht wird, ist die Implementierung eines Managementsystems, das den Anforderungen der EN ISO/IEC 17025 „Allgemeine Anforderungen an die Kompetenz von Prüf- und Kalibrierlaboratorien“ [2] entspricht, das mittelfristige Ziel der gemeinsamen Laborleitung. Nur so kann langfristig ein qualitativ hochwertiges und reproduzierbares, aber auch sicheres Arbeiten im Labor gewährleistet werden.

Neben der Unterstützung wissenschaftlicher Lehrstuhlmitarbeiter in den Bereichen Auftrags- und Antragsforschung sowie unterschiedlichen Aufgabenstellungen aus der Industrie- und Wirtschaft liegt eine der Hauptaufgaben der Laboraktivitäten in der studentischen sowie postakademischen Aus- und Weiterbildung. Diese Tatsache unterscheidet die Prüfstätten im IZR wesentlich von gängigen akkreditierten Prüf- und Kalibrierlaboratorien und fordert ein Qualitätsmanagementsystem, das einerseits diesen unterschiedlichen Aufgabenbereichen Rechnung tragen muss, andererseits aber auch Untersuchungsprozesse, die in situ - also direkt in den Betrieben - oder auf externen Prüfanlagen stattfinden, berücksichtigen soll.

\section{Aktuelle Infrastruktur zur Ermittlung von Gesteins- bzw. Materialkennwerten}

Derzeit können in den Laborräumlichkeiten des IZR normgerechte Untersuchungen an Fest- und an Lockergestein durchgeführt werden, aber auch gesteinsähnliche Werkstoffe, wie z. B. Beton oder Versatzmaterial sowie Bodenproben können bezüglich definierter Materialkennwerte analysiert werden. Spezialuntersuchungen sind ebenso möglich wie die Entwicklung und Ausarbeitung individueller Prüfverfahren.

\subsection{Probentransport}

Die untertägigen Laborräumlichkeiten der zwei Lehrstühle sind neben einem Personenlift und zwei Stiegenhäuser auch über einen Schwerlastenlift (Maximallast $6300 \mathrm{~kg}$ )
Abb. 1: Kernbohrsystem DD_750 HY

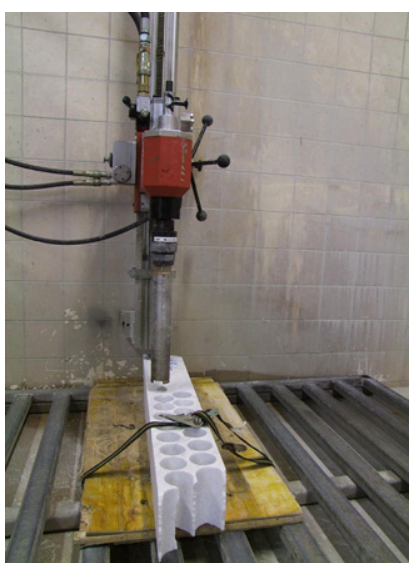

aufgeschlossen, sodass auch große Probenkörper bzw. schweres Material problemlos in die Untergeschosse des IZR transportiert werden können.

Zum internen Materialtransport stehen dem Laborpersonal weiters ein deichselgeführter Hubstapler der Marke Jungheinrich EJC 214 mit einer Nenntragfähigkeit von $1400 \mathrm{~kg}$ und ein Elektrogabelstapler der Marke Hyster J1.75XL mit einer Nenntragfähigkeit von $1750 \mathrm{~kg}$ zur Verfügung. Das Gesteinslabor, in dem vorwiegend Festgesteinsproben für Materialprüfungen vorbereitet werden, ist mit einem Leichtbaukransystem der Marke Konecranes (Traglast $1000 \mathrm{~kg}$ ) ausgestattet, sodass die maximalen Ausgangsprobengrößen vorwiegend von den Lastkapazitäten der Fördermittel limitiert werden.

\subsection{Probenvorbereitung}

Die Untersuchung von Festgestein beginnt prinzipiell mit der Probenvorbereitung, deren Ausführung von den weiterführenden Analysemethoden definiert wird. Da die Probenvorbereitung die Grundlage für qualitativ hochwertige Untersuchungsergebnisse darstellt, wird diese mit größter Sorgfalt ausgeführt und dokumentiert.

Als erster Schritt der Probenvorbereitung wird das Eingangsmaterial - egal ob dieses als Bohrkern oder Gesteinsblock vorliegt - in das Probendokumentationssystem aufgenommen, mit einer fortlaufenden Nummer versehen und fotografiert.

Liegt das Eingangsmaterial als Gesteinsblock vor, werden aus diesem Kerne gebohrt. Dies geschieht entweder mit einem Diamant-Kernbohrsystem DD-80 E der Firma Hilti [3] - Bohrbereich von 8 bis $82 \mathrm{~mm}$ Durchmesser - oder einem Diamant-Kernbohrsystem DD-750 HY (ebenfalls Hilti), das einen Bohrbereich von 52 bis 750 mm Durchmesser aufweist ([4]; vgl. Abb. 1).

Bilden Bohrkerne das Ausgangsmaterial für Analysen, wird als erster Untersuchungsschritt - falls erforderlich der ROD-Wert bestimmt, bevor das Probenmaterial weiter manipuliert wird, sprich die Kerne abgelängt und ihre Stirnseiten planparallel gefräst werden.

Zur Ablängung der Bohrkerne stehen den Labormitarbeitern zwei Steintrennmaschinen der Firma Gölz zur Verfügung. Für die Maschine vom Typ ST $100 \mathrm{~A}$ wird vom 


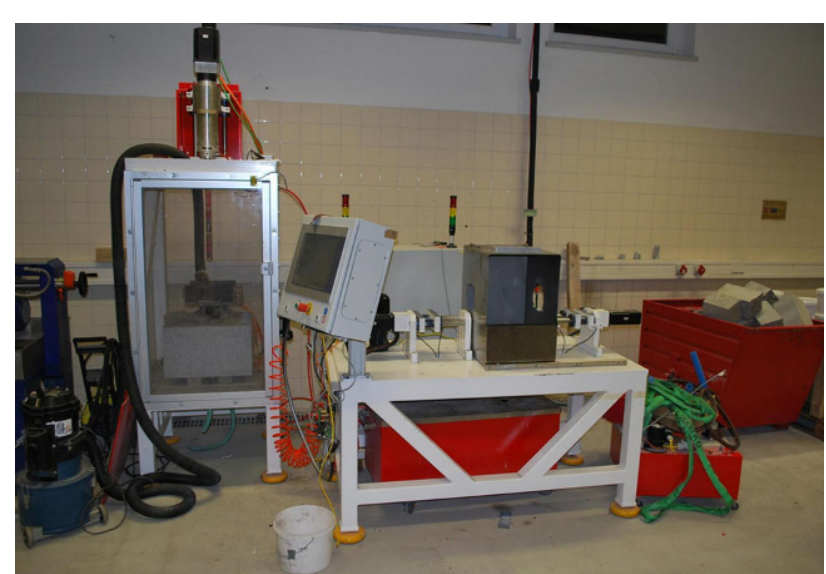

Abb. 2: Gesteinsverarbeitungssystem

Hersteller eine maximale Schnitttiefe von $110 \mathrm{~mm}$ und eine Schnittlänge von $1000 \mathrm{~mm}$ ausgewiesen [5]; für die Maschine vom Typ MS 700 A eine maximale Schnitttiefe von $280 \mathrm{~mm}$ und eine maximale Schnittlänge (bei einer Arbeitshöhe von $50 \mathrm{~mm}$ ) von $800 \mathrm{~mm}$ [6].

Mit einer Planschleifmaschine vom Typ 305 der Firma Strassentest Baustoff-Prüfsysteme erfolgt das Planschleifen der Prüfkörper, die von beiden Seiten gleichzeitig geschliffen werden, sodass die Stirnseiten der Proben planparallel hergestellt werden können [7].

Zukünftig wird dem Laborpersonal zur Probenvorbereitung auch ein hausintern entwickeltes und gebautes Gesteinsbearbeitungssystem zur Verfügung stehen, bei dem mittels zentraler CNC-Steuerung von einer Person zwei Prozesse (Kernbohren und Ablängen/Planschleifen) gleichzeitig ausgeführt werden können. Durch das neue System soll einerseits die Qualität der Probenkörper erhöht, andererseits aber auch die Prüfkörperherstellung bezüglich Arbeitssicherheit verbessert werden ([8]; vgl. Abb. 2).

Nach der Probenherstellung wird die Geometrie messtechnisch erfasst und dokumentiert sowie eine Massebestimmung durchgeführt. Die Proben erhalten eine eindeutige Bezeichnung, die auch die fortlaufende Eingangsnummer beinhaltet.

Für Feuchtebestimmungen können die Gesteinsproben einem Trocknungsprozess unterzogen werden. Dafür stehen im Labor ein multifunktionaler Wärme- bzw. Trockenschrank mit forcierter Umluft und Timer vom Typ FED und ein Klimaschrank für konstante Bedingungen mit Programmregelung vom Typ KBF der Firma Binder sowie ein Trockenschrank der Firma Heraeus (Typ T 5042 E) zur Verfügung.

\subsection{Zerstörungsfreie Festgesteinsprüfung}

Bevor die Probenkörper durch Druck- oder Zugprüfungen zerstört werden, werden diese routinemäßig durchschallt, sodass die Ausbreitungsgeschwindigkeiten von $\mathrm{p}$ - und s-Wellen im untersuchten Gestein ermittelt werden und

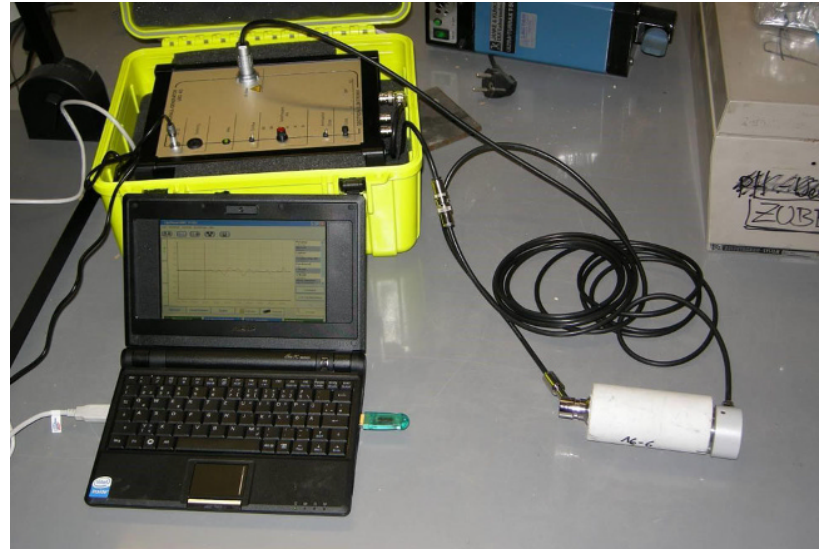

Abb. 3: Ultraschallmesssystem UKS-D

folglich elastische Materialkennwerte - wie z. B. der dynamische E-Modul - bestimmt werden können.

Hier kommt das PC-gestützte Ultraschall-Messsystem UKS-D der Firma Geotron Elektronik zum Einsatz, das mit einem USG 40 Ultraschall-Generator und dem PCOszilloskop PicoScope ${ }^{\circledR}$ ausgestattet ist und mit unterschiedlichen Prüfköpfen versehen werden kann ([9], vgl. Abb. 3).

\subsection{Zerstörende Festgesteinsprüfung}

Für Druck- und Zugversuche an Prüfkörpern steht den Wissenschaftlern und Forschern der Lehrstühle für Subsurface Engineering und Bergbau, Bergtechnik und Bergwirtschaft eine Servohydraulische Gesteinsprüfpresse Typ MTS 815 zur Verfügung. Diese Prüfmaschine ist ideal für ein- und triaxiale Druckversuche sowie für direkte und indirekte Zugversuche an Gesteinsproben ausgelegt und erreicht dafür in axialer Richtung Druckkräfte bis zu 2850 kN und Zugkräfte bis $1340 \mathrm{kN}$ ([10]; vgl. Abb. 4).

Abb. 4: Gesteinsprüfpresse Typ MTS 815

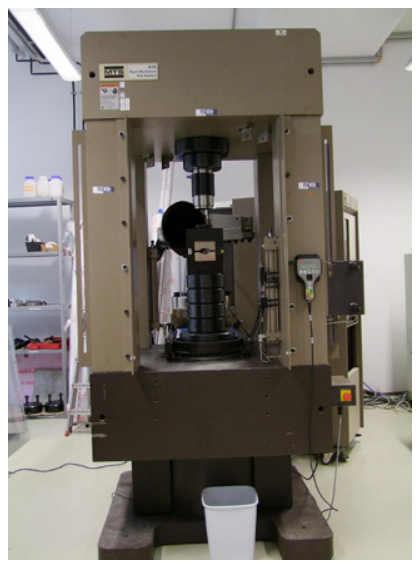


Abb. 5: Statische und dynamische Universalprüfmaschine Typ UL01-SH0100-S1
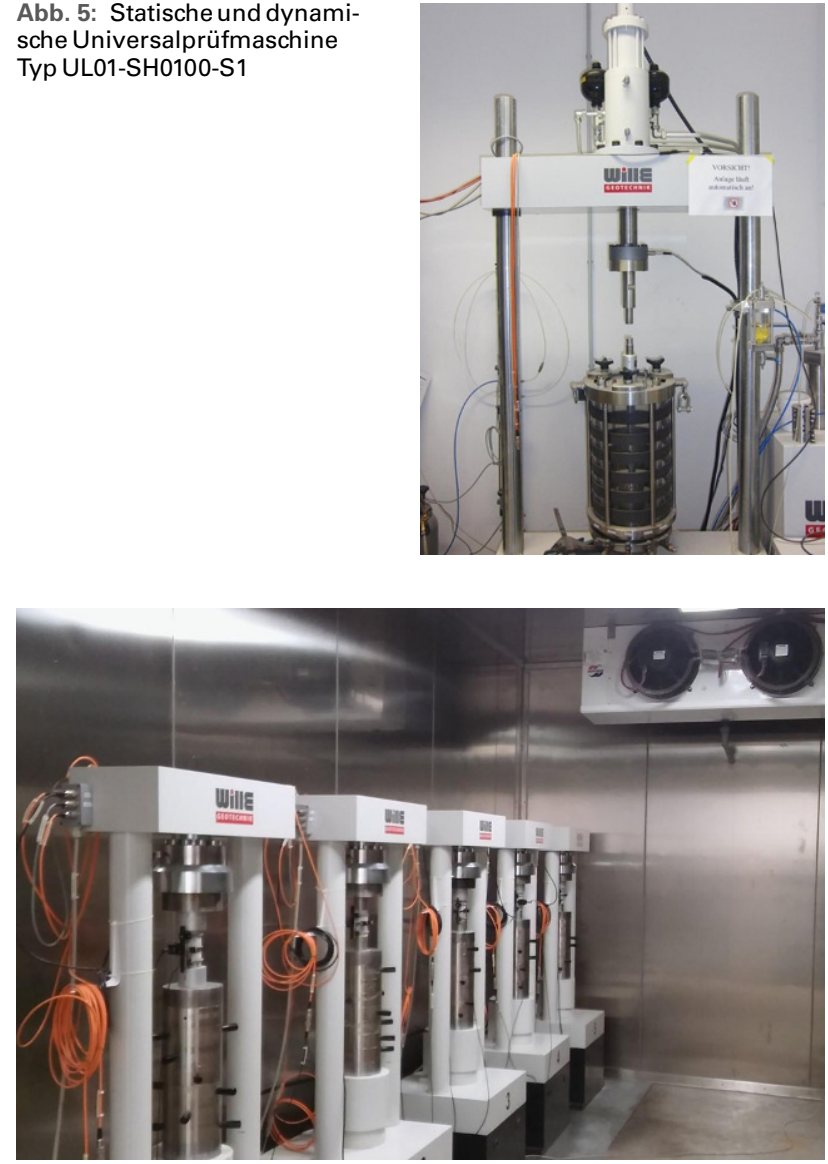

Abb. 6: Klimakammer mit Langzeitprüfständen vom Typ Wille Geotechnik UL-300

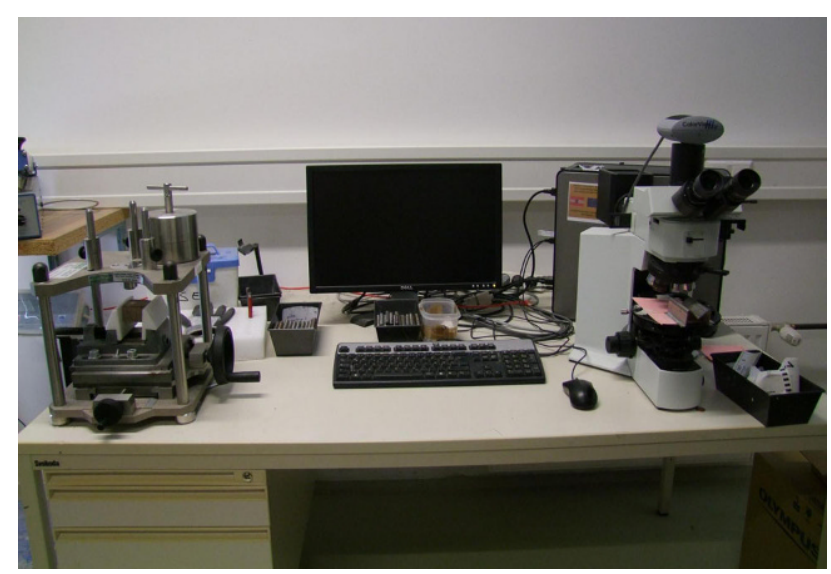

Abb. 7: Cerchar Rock Abrasiveness Tester

\subsection{Untersuchung von Boden- und Versatzmaterial}

Untersuchungen an Boden- oder Versatzmaterial können im IZR mit einer statischen und dynamischen Universalprüfmaschine vom Typ UL01-SH0100-S1 des Produzenten Wille Geotechnik in Form von Triaxialprüfungen durchgeführt werden. Die Prüfmaschine ist für die Durchführung von statischen und dynamischen Zug- und Druckbeanspru-
Abb. 8: Abrasimeter für LCPC Prüfungen

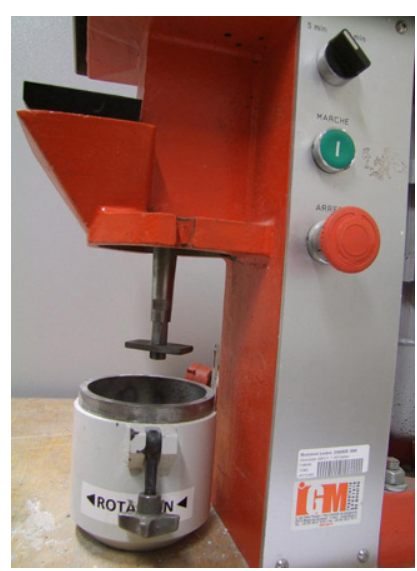

chungen im Schwell- und Wechselbereich konzipiert, und mit ihr kann eine maximale statische Prüfkraft von $100 \mathrm{kN}$ und eine dynamische Prüflast von $80 \mathrm{kN}$ realisiert werden ([11]; vgl. Abb. 5).

\subsection{Untersuchung von Langzeiteigenschaften mineralischer Rohstoffe und gesteins- ähnlicher Werkstoffe}

Um das Langzeitverhalten von mineralischen Rohstoffen und gesteinsähnlichen Werkstoffen untersuchen zu können, wurde vom Lehrstuhl für Subsurface Engineering im IZR eine Klimakammer mit einer Fläche von $18 \mathrm{~m}^{2}$ und einem Volumen von $52,5 \mathrm{~m}^{3}$ installiert. Die möglichen Temperaturbereiche erstrecken sich von -10 bis $+80^{\circ} \mathrm{C}$ und die relative Luftfeuchtigkeit kann zwischen 10 und $90 \%$ reguliert werden.

Die Kammer ist mit fünf Langzeit-Kriechprüfständen vom Typ UL-300 der Firma Wille Geotechnik ausgestattet, auf denen Proben einaxial belastet werden können. Die Prüfstände sind für eine maximale statische Prüflast von $300 \mathrm{kN}$ ausgelegt und können sowohl kraft- wie auch verformungsgesteuert geregelt werden ([12]; vgl. Abb. 6).

\subsection{Bestimmung der Abrasivität von Fest- und Lockergestein}

Mit einem Cerchar Rock Abrasiveness Tester der britischen Firma Ergotech Ltd. kann im IZR die Abrasivität von Festgesteinen bestimmt werden, die durch den Cerchar Abrasivitäts Index (CAl) ausgedrückt wird.

Die Bauart des Testers entspricht dem „West-Gerät“, dessen Anwendung sich für Gesteinsarten, die in Mitteleuropa vorherrschen, bewährt hat; als Prüfstifte werden entweder Stifte der Härte HRC 40-43 oder HRC 54-56 verwendet. Die Auswertung der Prüfstifte erfolgt unter einem Mikroskop durch ein computerunterstütztes Bildverarbeitungsprogramm (vgl. Abb. 7).

Mit dem LCPC (Laboratoire Central des Ponts et Chaussées) Test wird im Labor die Abrasivität von Lockermaterial bestimmt. Im IZR wird dieser Test mit einem Abrasimeter 
Abb. 9: Los Angeles Maschine 4320
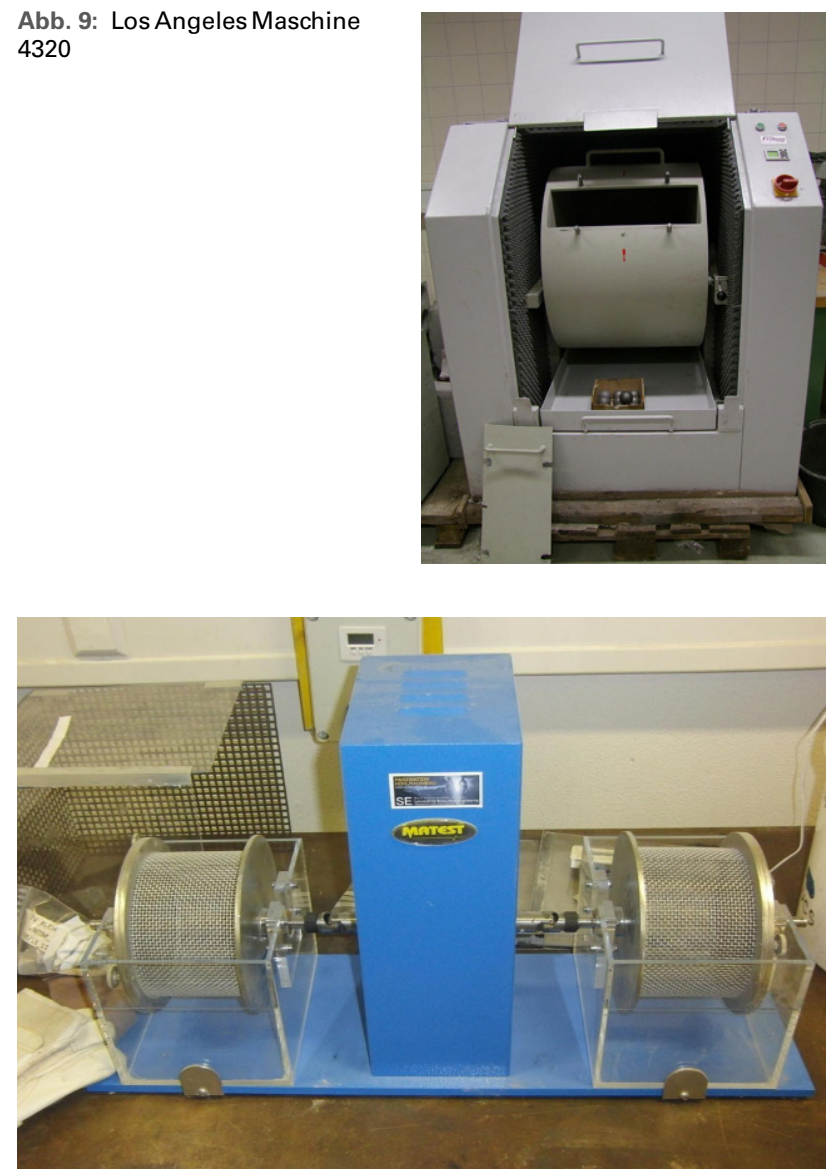

Abb. 10: A130 Slake Durability Apparatus

Abb. 11: Viskomat NT

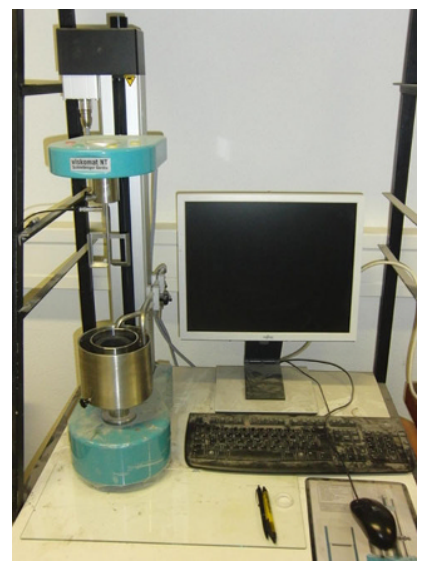

der französischen Firma ICM nach dem französischen Standard NF P18-579 durchgeführt [13]. Da für diesen Test die Korngrößenverteilung zwischen 4 und 6,3 mm liegen muss, muss das Prüfmaterial einer Siebung unterzogen werden. Dabei können die Laboranten im Impulszentrum für Rohstoffe auf eine Analyse Siebmaschine AS 300 control von Retsch-Verder zurückgreifen ([14]; vgl. Abb. 8).

\subsection{Bestimmung des Widerstands gegen Zertrümmerung von Gesteinskörnungen}

Eine Los Angeles Maschine 4320 der Firma Fröwag GmbH dient den Lehrstühlen als Prüfeinrichtung nach EN 1097-2 [15] und EN 13450 [16] zur Bestimmung des Widerstands gegen Zertrümmerung mit dem Los Angeles-Prüfverfahren ([17]; vgl. Abb. 9).

\subsection{Bestimmung der Zerfallsbeständigkeit von Gestein}

Mit Hilfe des Siebtrommelversuchs wird Gestein hinsichtlich seiner Resistenz gegen mechanischen Abrieb klassifiziert. Für die Versuchsdurchführung, die nach Empfehlung Nr. 20 des Arbeitskreises 3.3 "Versuchstechnik Fels" der Deutschen Gesellschaft für Geotechnik e. V.: Zerfallsbeständigkeit von Gestein - Siebtrommelversuch [18] durchgeführt wird, steht als Analysegerät der A130 Slake Durability Apparatus der Firma Matest zur Verfügung ([19]; vgl. Abb. 10).

\subsection{Viskositätsbestimmung}

Für Viskositätsbestimmungen steht den Labormitarbeitern mit dem Viskomat NT der Firma Schleibinger Geräte Teubert u. Greim $\mathrm{GmbH}$ ein modernes und leistungsfähiges Analysegerät zur Verfügung (vgl. Abb. 11).

\section{Betrieb der Prüfanlagen und Analysegeräte}

Sämtliche Laboranten sind an den Prüfanlagen und Analysegeräten ausgebildet und mit den standardisierten Prüfverfahren vertraut, sodass diesbezüglich Prüfaufträge rasch und qualitativ hochwertig abgewickelt werden können.

Spezialanwendungen und die Ausarbeitung individueller Prüfverfahren werden von wissenschaftlichen Mitarbeitern der Lehrstühle durchgeführt, die dabei vom Laborpersonal bestmöglich unterstützt werden.

Studenten und Auszubildende werden vom Laborpersonal anhand der Arbeits- und Prüfanweisungen in den Prüfprozessen unterwiesen, sodass in jeder Hinsicht ein sicheres Arbeiten im Labor gewährleistet ist.

Funding. Open access funding provided by Montanuniversität Leoben.

Open Access Dieser Artikel wird unter der Creative Commons Namensnennung 4.0 International Lizenz (http://creativecommons.org/licenses/ by/4.0/deed.de) veröffentlicht, welche die Nutzung, Vervielfältigung,

Bearbeitung, Verbreitung und Wiedergabe in jeglichem Medium und Format erlaubt, sofern Sie den/die ursprünglichen Autor(en) und die Quelle ordnungsgemäß nennen, einen Link zur Creative Commons Lizenz beifügen und angeben, ob Änderungen vorgenommen wurden. 


\section{Literatur}

1. Kaltenbeck, B.: Die neue Welt der Rohstoffe eröffnet in Leoben, Medieninformation der Steirischen Wirtschaftsförderung (SFS), Leoben, 2011

2. ÖNORM EN ISO/IEC 17027 Allgemeine Anforderungen an die Kompetenz von Prüf- und Kalibrierlaboratorien, Ausgabe 2007-01-01

3. Bedienungsanleitung Hydraulisches Diamant-Kernbohrsystem DD750 HY, Hilti Corporation, Schaan (FL)

4. Bedienungsanleitung Hydraulisches Diamant-Kernbohrsystem DD$80 \mathrm{E}$, Hilti Corporation, Schaan (FL)

5. Bedienungsanleitung Steintrennmaschine Typ ST 100 A, Gölz $\mathrm{GmbH}$, Hellenthal-Blumenthal (D)

6. Bedienungsanleitung Steintrennmaschine Typ MS 700 A, Gölz $\mathrm{GmbH}$, Hellenthal-Blumenthal (D)

7. Bedienungsanleitung Planschleifmaschine Typ 305, Strassentest Baustoff-Prüfsysteme OHG, Aschaffenburg (D)

8. Hohl, W.: Gesteinsproben; Triple M Zeitschrift der Montanuniversität Leoben, Ausgabe 4/2016, p 10

9. Installations- und Bedienungshandbuch Handbuch LightHouse UMPC, Version 1.0, Geotron-Elektronik, Pirna (D), 2008

10. MTS Rock and Concrete Mechanics Testing System, Technical Description, MTS, Eden Prairie (USA)
11. Betriebsanleitung Statische und dynamische Universalprüfmaschine Typ UL01-SH0100-S1, Wille Geotechnik, Rosdorf/Göttingen (D), 2011

12. Bedienungsanleitung Kriechprüfstand UL-300, Wille Geotechnik, Rosdorf/Göttingen (D), 2013

13. Bedienungsanleitung Abrasimeter, IGM, Magny les Hameaux (F)

14. Bedienungsanleitung Analyse-Siebmaschine Typ AS 300 control, Retsch $\mathrm{GmbH}$, Haan (D)

15. DIN EN 1097-2 Prüfverfahren für mechanische und physikalische Eigenschaften von Gesteinskörnungen - Teil 2: Verfahren zur Bestimmung des Widerstandes gegen Zertrümmerung, Ausgabe 2010-07

16. DIN EN 13450 Gesteinskörnungen für Gleisschotter, Ausgabe 200306

17. Bedienungsanleitung Los Angeles Maschine 4.320, Fröhlich und Wagner GmbH Prüfanlagen für Baustoffe und Umwelt, Obersulm/ Eschenau (D)

18. Herzl, P.: Empfehlung Nr. 20 des Arbeitskreises 3.3 „Versuchstechnik Fels" der Deutschen Gesellschaft für Geotechnik e.V.: Zerfallsbeständigkeit von Gestein - Siebtrommelversuch, Bautechnik 79 (2002), Heft 2, pp 101-105

19. Manual A130 Slake Durability Apparatus, Matest Material Testing Equipment, Treviolo (I) 